\title{
Effect of Hope Promotion Cognitive Training on Characteristic Abilities, Psychological Flexibility, and Cognitive Functions Reconstruction of Veterans' Wives
}

\section{ART ICLE INF O}

\section{Article Type}

Original Research

\section{Authors}

Abbasi M.*PhD,

Pirani Z. ${ }^{1} P h D$,

Zanguei A.R. ${ }^{1} M S C$,

Zarei M.H. ${ }^{2} B A$

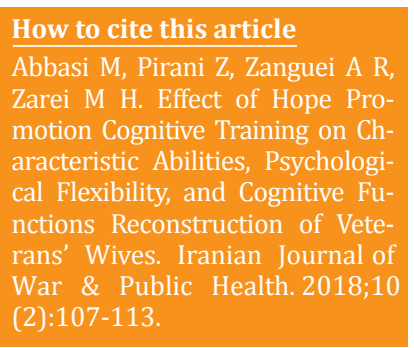

*Psychology Department, Literature \& Human Sciences Faculty, Salman Farsi University of Kazerun, Kazerun, Iran

${ }^{1}$ Psychology Department, Human Sciences Faculty, Arak Branch, Islamic Azad University, Arak, Iran ${ }^{2}$ Psychology Department, Literature \& Human Sciences Faculty, Salman Farsi University, Kazerun, Iran

\section{Correspondence}

Address: Students Affair Deputy, Salman Farsi University, First of Taleqani Street, Ershad Junction, Kazerun, Iran

Phone: +98 (71) 42226050

Fax: +98 (71) 42226050

abbasi@kazerunsfu.ac.ir

\section{Article History}

Received: August 4, 2017

Accepted: January 15, 2018

ePublished: April 10, 2018

\section{A B S T R A C T}

Aims It seems that the cognitive and characteristic aspects of some of the veterans' wives have been influenced by the consequences of war on their lives. The aim of this study was to evaluate the effect of hope promotion cognitive training on characteristic abilities, psychological flexibility, and cognitive functions reconstruction of veterans' wives.

Materials \& Methods In this semi-experimental study with pre-test and post-test design with control group, 50 veterans' wives from Kazeroon, Iran in 2017 were selected by available sampling method; they were randomly divided to two experimental $(n=25)$ and control $(n=25)$ groups. To collect the data, Cognitive Abilities Questionnaire, Acceptance and Action Questionnaire-II (AAQ-II), and Values in Action Inventory were used. The experimental group received cognitive hope promotion training for 6 weeks. Data analysis was performed by SPSS 23 software, using multivariate analysis of covariance analysis.

Findings By controlling the effects of pre-test, there was a significant difference between the experimental and control groups in terms of overall score of characteristic abilities, psychological flexibility, and cognitive functions reconstruction $(p=0.0001)$. Also, after the intervention, the mean scores of characteristic abilities and cognitive functions reconstruction increased significantly in the experimental group $(\mathrm{p}<0.05)$; these increases were not significant only in inhibitory control and selective attention as well as social cognition ( $p>0.05)$.

Conclusion Hope promotion cognitive training has an effect on the characteristic abilities, psychological flexibility, and cognitive functions reconstruction of veterans' wives, but it does not affect the inhibitory control and selective attention as well as social cognition.

Keywords Hope Promotion Cognitive Training; Characteristic Abilities; Psychological Flexibility; Cognitive Functions; Veterans

\section{CIT A T I O N L I N KS}

[1] Military-related post-traumatic stress disorder ... [2] Correlation of social support with social ... [3] Comparitive study of some mental ... [4] The relationship between depression and how ... [5] Executive function and neuropsychological ... [6] The effect of cognitive rehabilitation ... [7] Neural evidence for dissociable components ... [8] The psychological (emotional and cognitive) ... [9] The effectiveness of acceptance and commitment ... [10] Acceptance and commitment therapy ... [11] Acceptance and commitment therapy ... [12] Does experientialavoidance mediate the effects of maladaptive coping styles ... [13] Is it the sound or your relationship ... [14] The investigate the relationship between ... [15] Role of character strengths and stress in psychological ... [16] Positive psychology progress: Empirical ... [17] Strengths of character, orientations to ... [18] The psychological and acmeological approach in an educational ... [19] Strengths of character and ... [20] The role of locus of control and coping style in predicting loungitudinal ... [21] Hope and the meaningful ... [22] The influence of hope on appraisals ... [23] The influence of between and within ... [24] The effectiveness of cognitive behavior ... [25] The effect of cognitive hope enhancing training ... [26] The effect of cognitive hope enhancing ... [27] Theoretical and practical research in the ... [28] Investigating the factor structure of students ... [29] Cognitive abilities questionnaire: Development ... [30] Hope theory, measurements, and ... [31] Hope, but not optimism predicts academic ... [32] Rumination and suicidal ideation: The moderating ... [33] An integrative review of adolescent ... [34] Hopein the context of lung cancer: Relationships of hope to symptoms and ... [35] Great expectations: A meta-analytic examination ... [36] Embracing your demons: An overview ... [37] Psychobiological mechanisms of resilience ... [38] The role of mindfulness and psychological flexibility in somatization, depression, anxiety, and general psychological distress in a nonclinical college ... 
ايجاد تنش در محيط خانواده خود مىشوند [3] و اين امر واقعنكرى

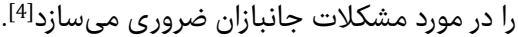

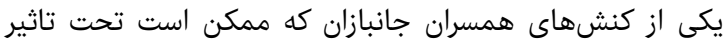

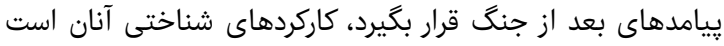

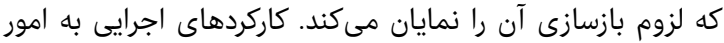

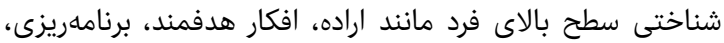

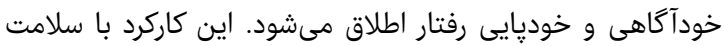

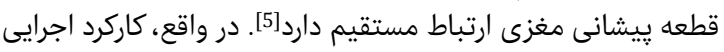

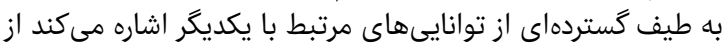

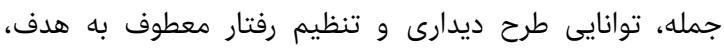

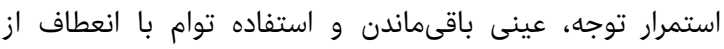

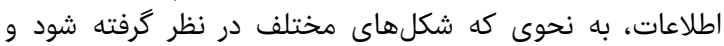

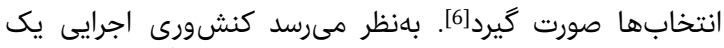

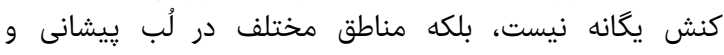

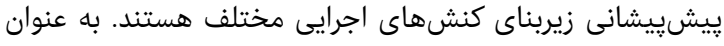

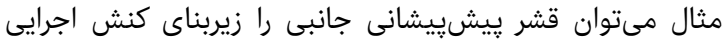

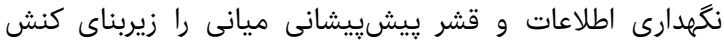

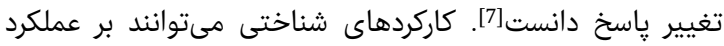

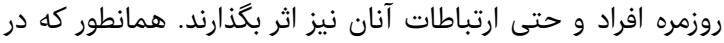

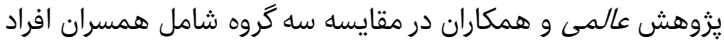

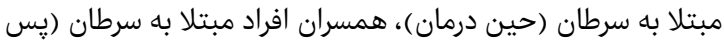

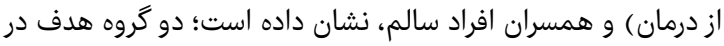

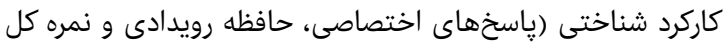

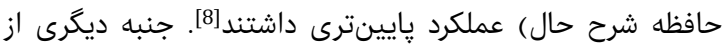

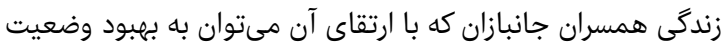

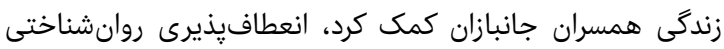

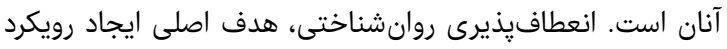

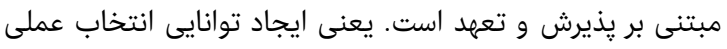

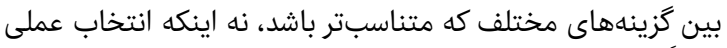

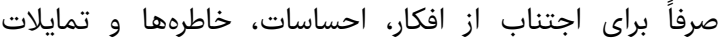

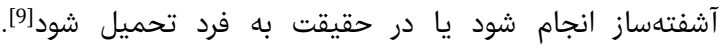

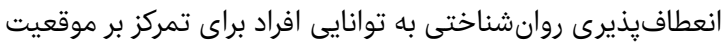

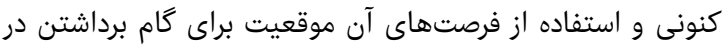

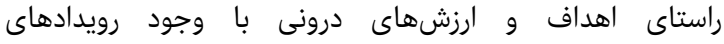

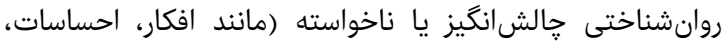

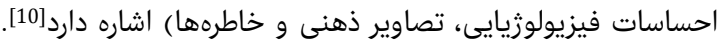

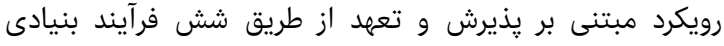

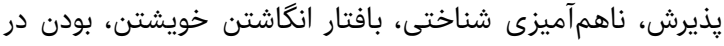

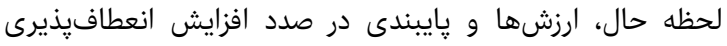

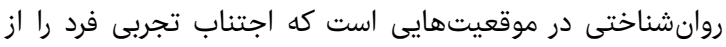

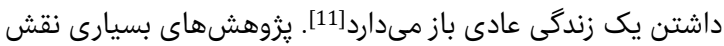

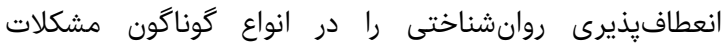

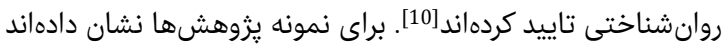

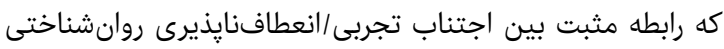

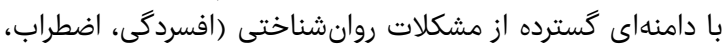

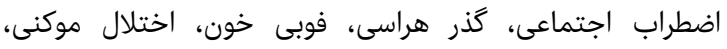

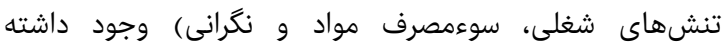

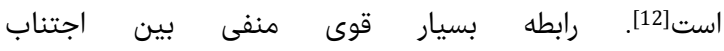
تجربى/انعطافنايذيرى روانشناختى، سلامت عمومى و و كيفيت زندكى وجود

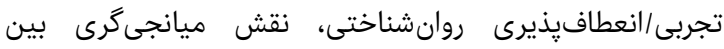

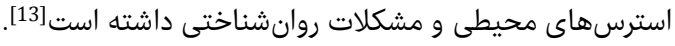

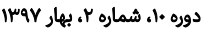

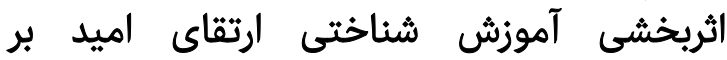

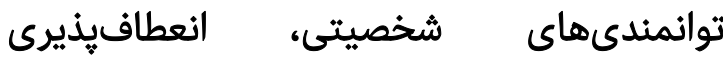

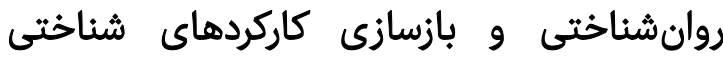 همسران جانبازان}

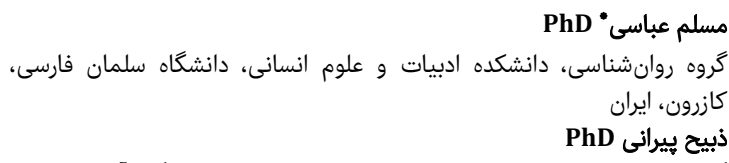

كروه روانشناسى، دانشكده علوم انسانى، واحد اراك، دانشكاه آزاد اسلامى،

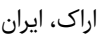

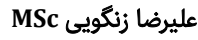

كَره روانشناسى، دانئ دانشكده علوم انسانى، واحد اراك، دانشكاه آزاد اسلامى،

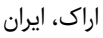

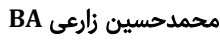

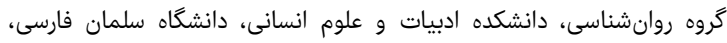

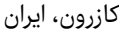

جكيده

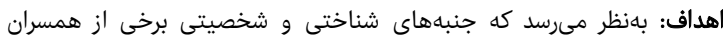

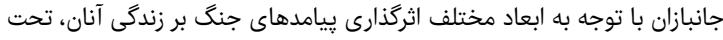

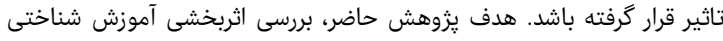

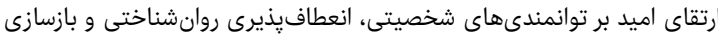
كاركرد شناختى همسران جانبازان بود إندان

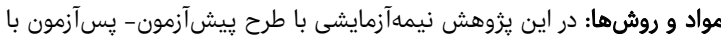

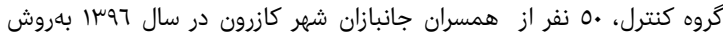

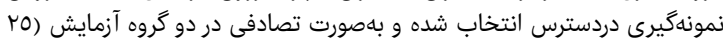

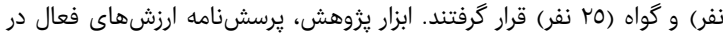

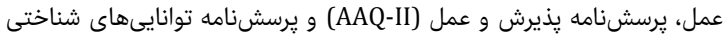

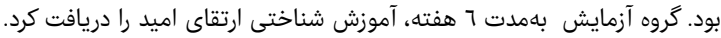

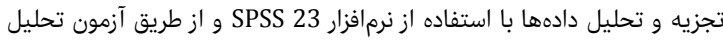

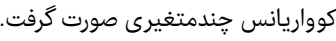

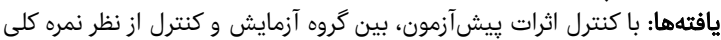

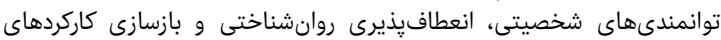

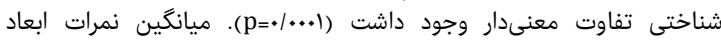

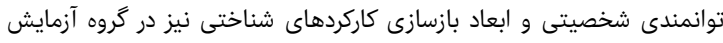

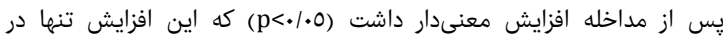

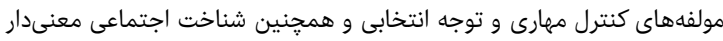

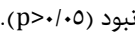

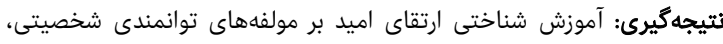

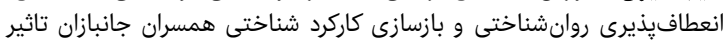

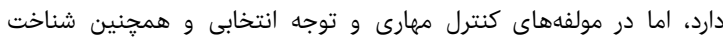

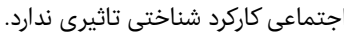

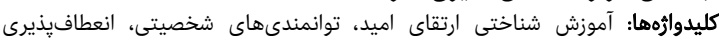
روانشناختى، كاركردهاى شناختى، جانبازان آمران

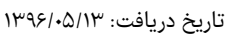

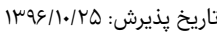

abbasi@kazerunsfu.ac.ir :نويسنده مسئول: تورل:

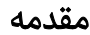

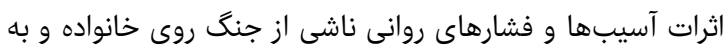

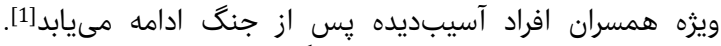

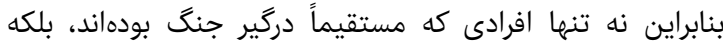

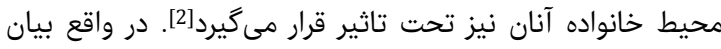

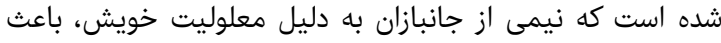


اثربخشى آموزش شناختى ارتقاى اميد بر توانمندىهاى شخصيتى، انعطافيذيرى روانشناختى و بازنازي كاركردهاى شناختى همسران جانبازان 1.9

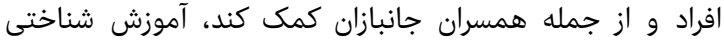

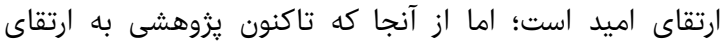

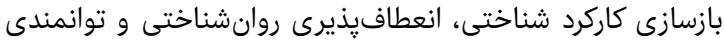

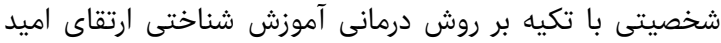

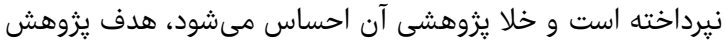

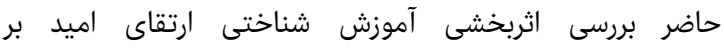

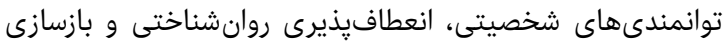
كاركرد شناختى همسران جانبازان بوده انعطافي

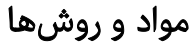

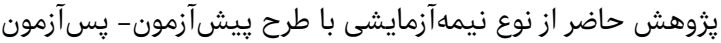

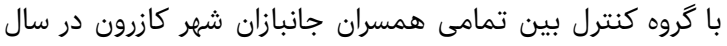

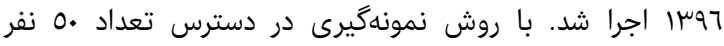

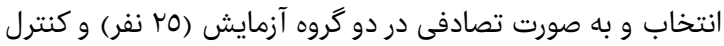

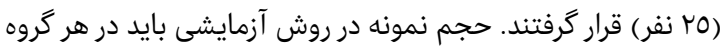

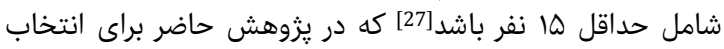

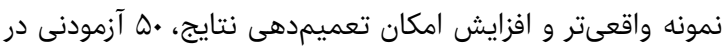

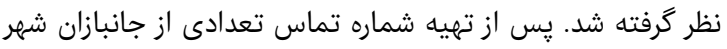

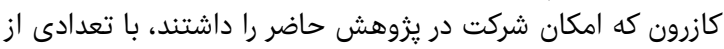

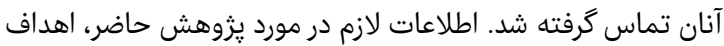

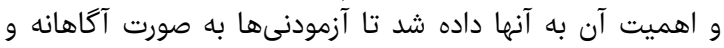

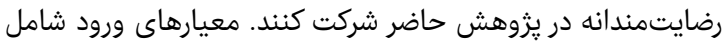

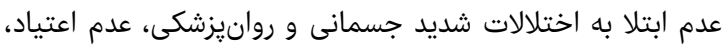

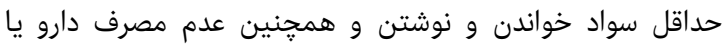

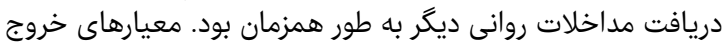

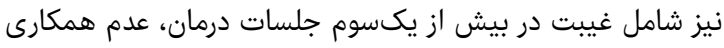

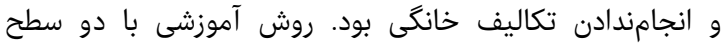

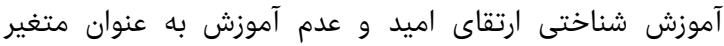

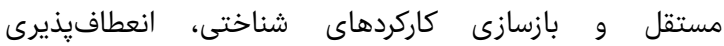
روانشناختى و توانمندى شخصيتى به عنوان شارى متغيرهاى

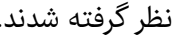

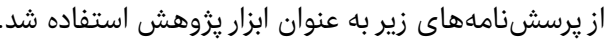

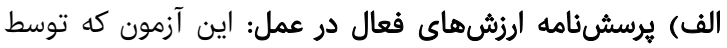

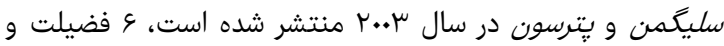

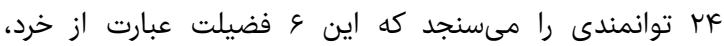

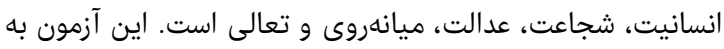

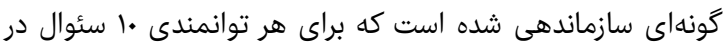

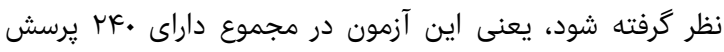

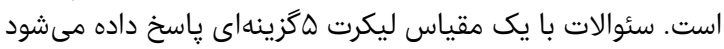

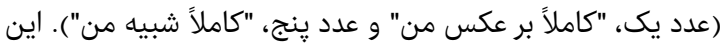

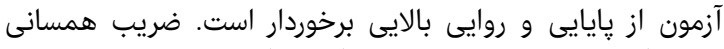

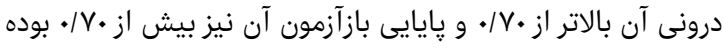

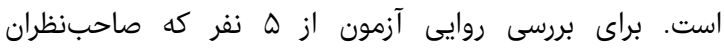

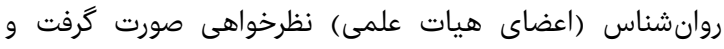

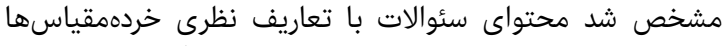

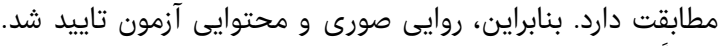

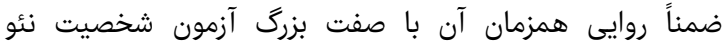

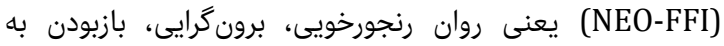

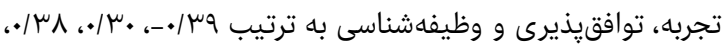

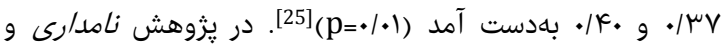
همكاران[25]، عدد آلفاى كرونباخ براى كل مقيت امدياس

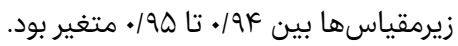

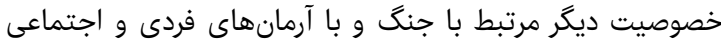

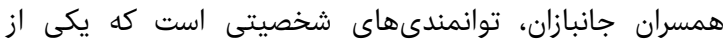

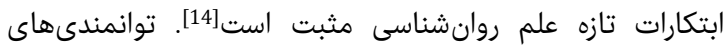

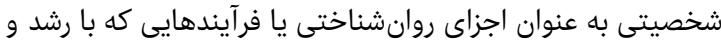

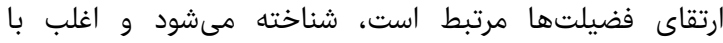

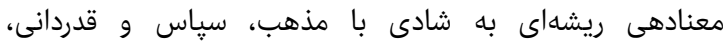

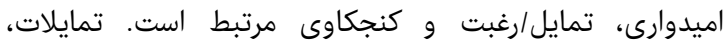

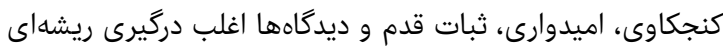

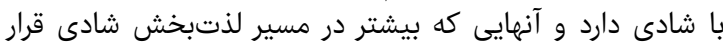

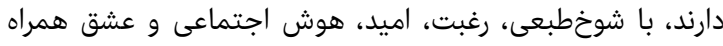

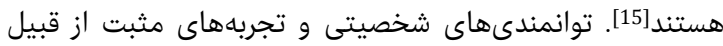

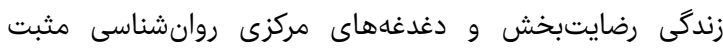

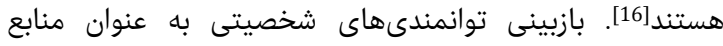

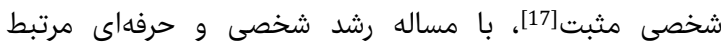

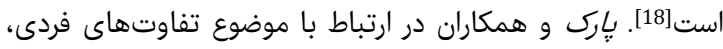

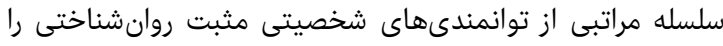

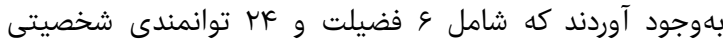
معين است. همجنين، نشان داده شده است كه برخى إنى از ابعاد

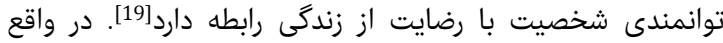
توانمندىهاى شخصيتى افراد را قادر مىسازيت زئد تا عملكرد بهترى

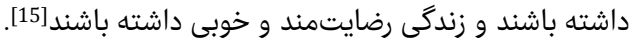

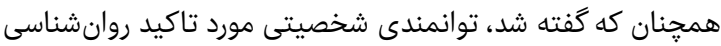

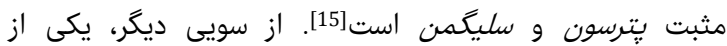

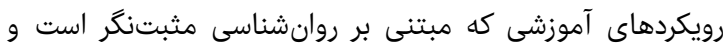

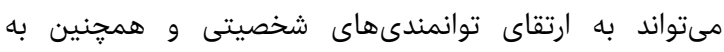

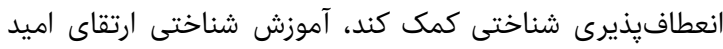

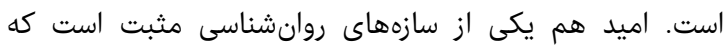

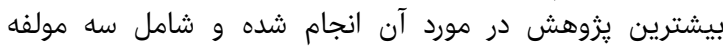

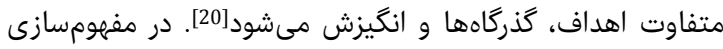

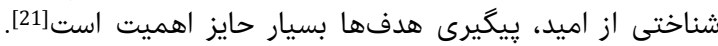

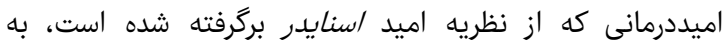

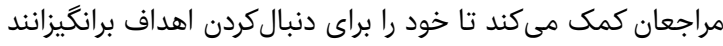

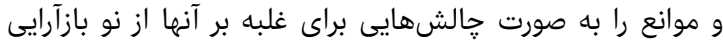

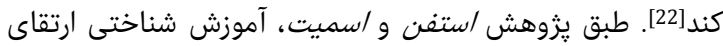

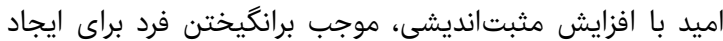

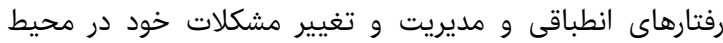

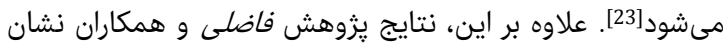

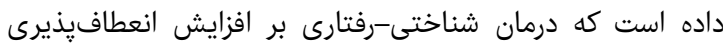

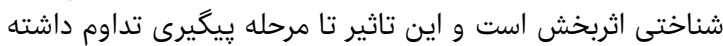

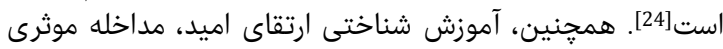

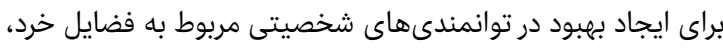

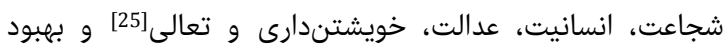

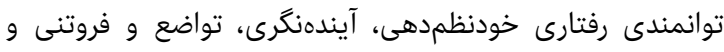

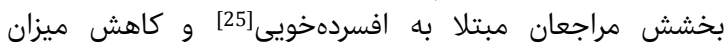

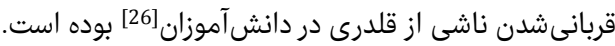

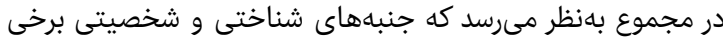

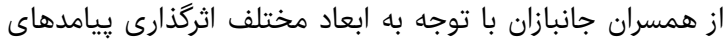

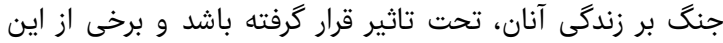

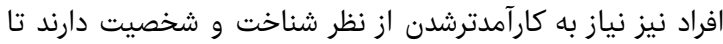

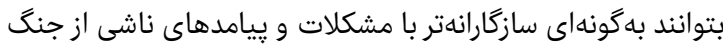

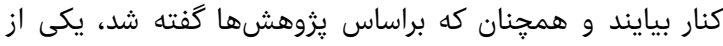

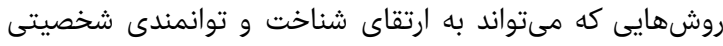


جدول () شرح جلسات يروتكل آموزشى شناختى ارتقاى اميد براساس الكوى

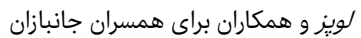

جلسه اول

تعيين نمره پايه: آشنايى آزمودنىها و درمانكَر با همديكر، تعيين نمره پايه متغيرهاى وابسته

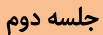

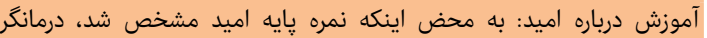

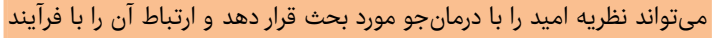

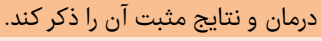
جلسه سوم إنمائ

سازماندهى اميد براى مراجع: در اين مرحله، مراجع فهرستى از عناصر و واجزاى

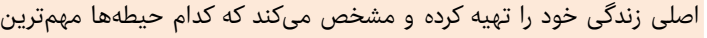

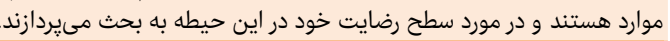

جلسه جهارم

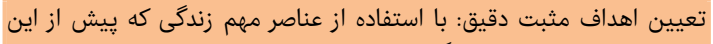

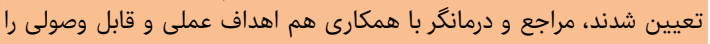

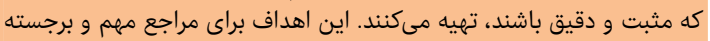

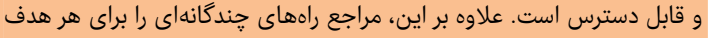

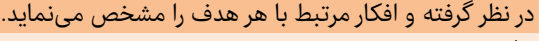
جلسه ينجم تجسم اهداف، تمرين كار را كامل مى كند: وقتى مراجع و درمانكانكر روى اين

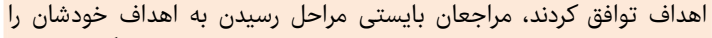

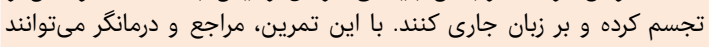

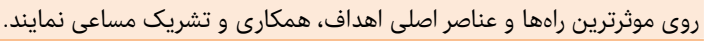

جلسه ششم مورتي

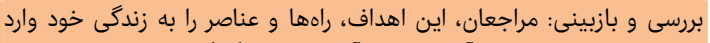

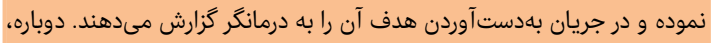

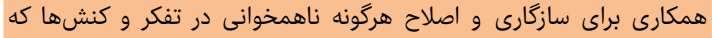

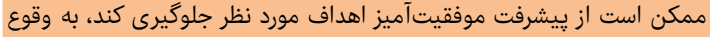

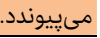

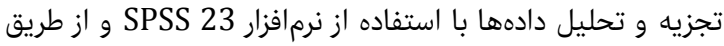

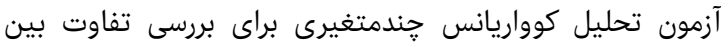

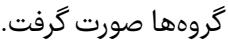

يافتهها با كنترل اثرات يِشآ آزمون، بين گروه آزمايش و كنترل از نظر نمره

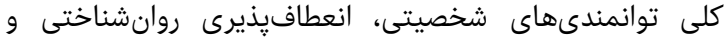

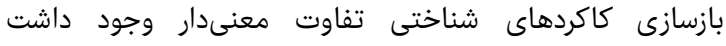

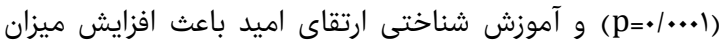

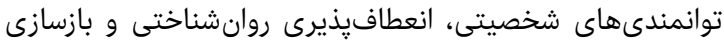

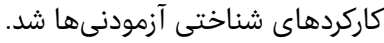

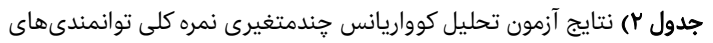

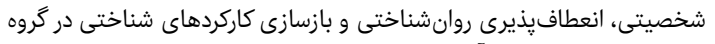

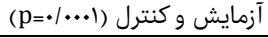

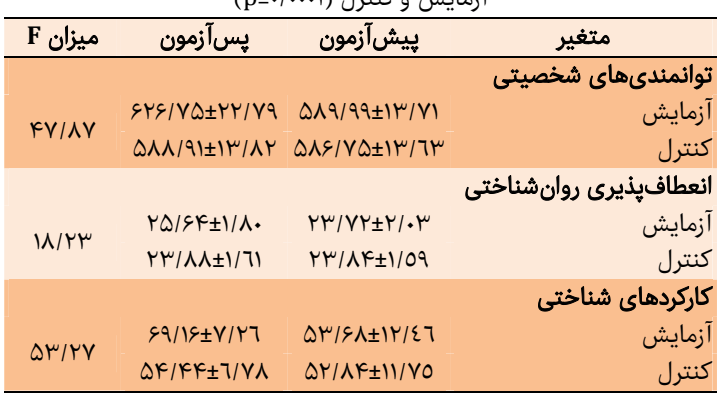

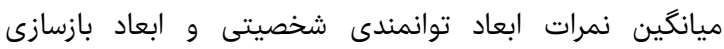
كاركردهاى شناختى در گروه آزمايش يس إن از مداخله افزايش

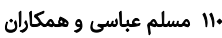

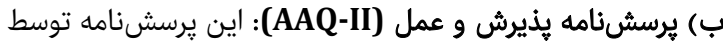

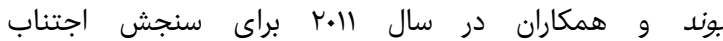

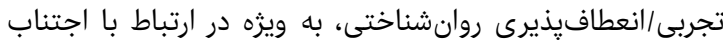

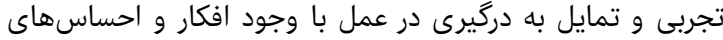

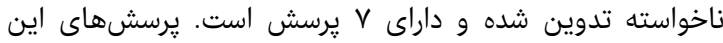

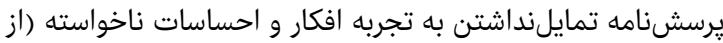

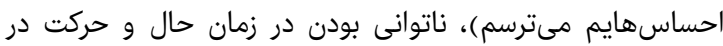

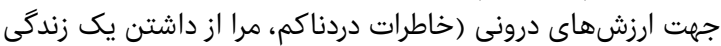

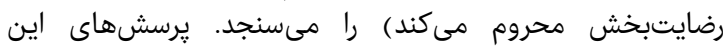

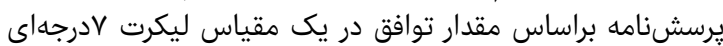

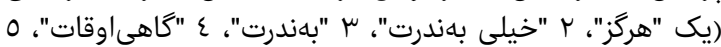

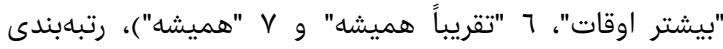

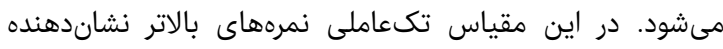

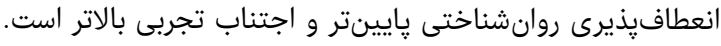

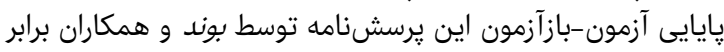

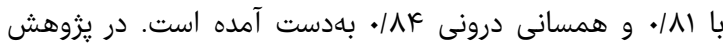

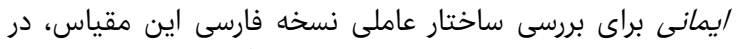

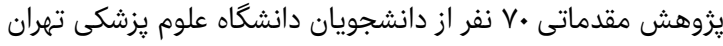

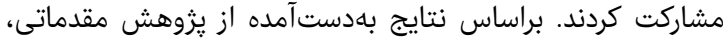

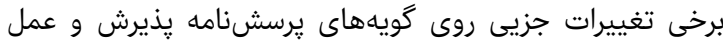

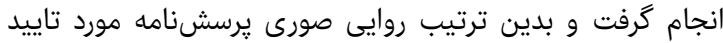

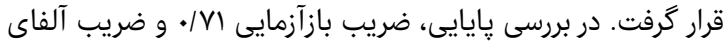

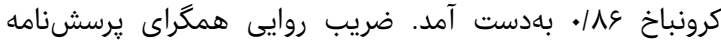

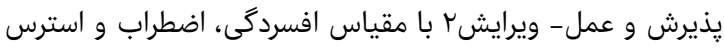

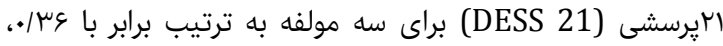

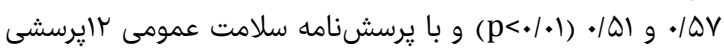

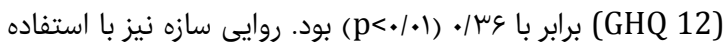

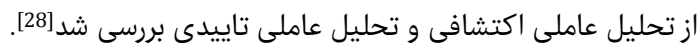

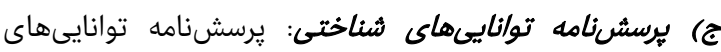

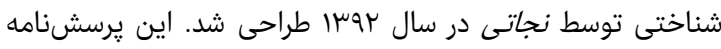

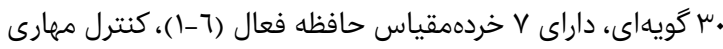

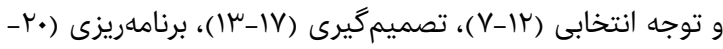

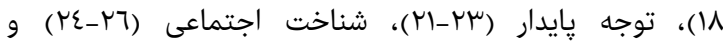

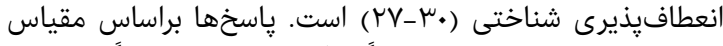

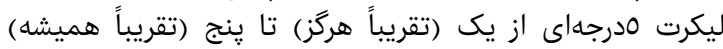

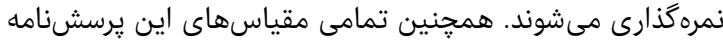

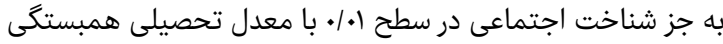

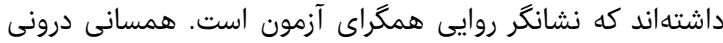

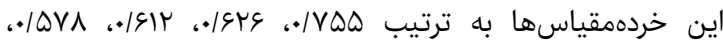

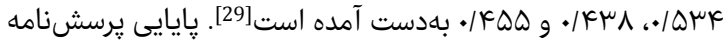

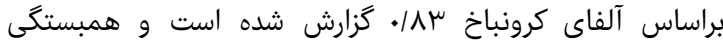

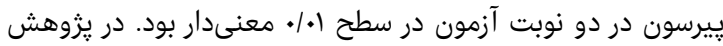

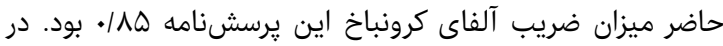

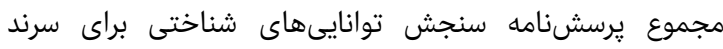

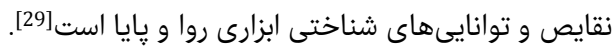

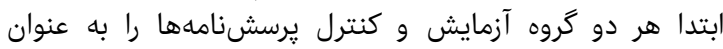

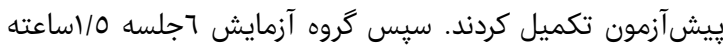

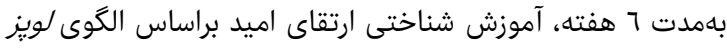

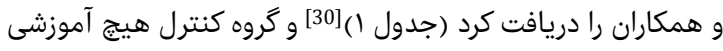

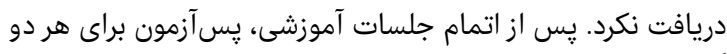

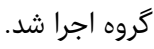




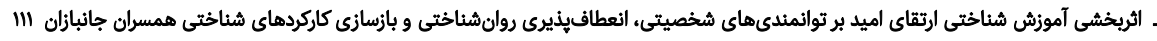

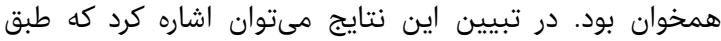

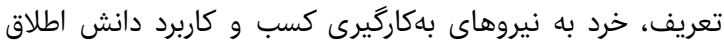

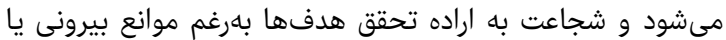

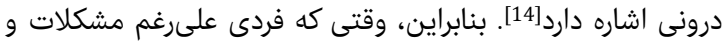

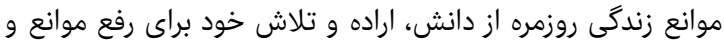

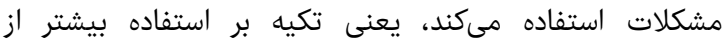

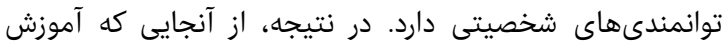

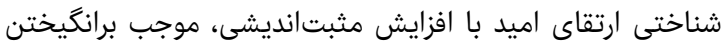

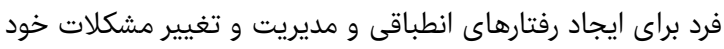

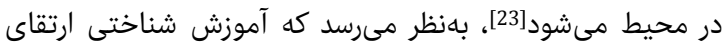

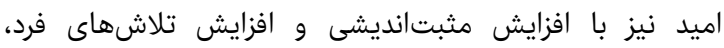

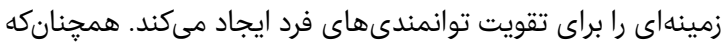

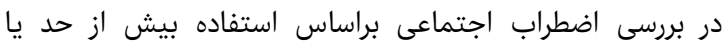

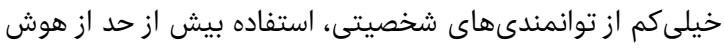

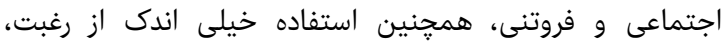

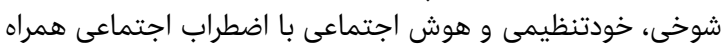

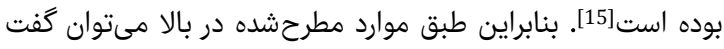

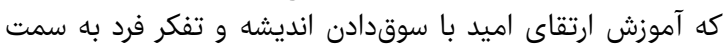

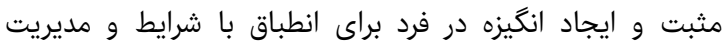

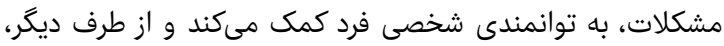

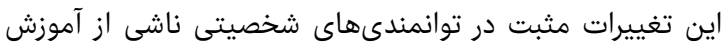

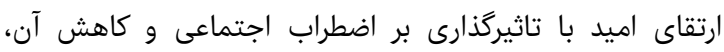

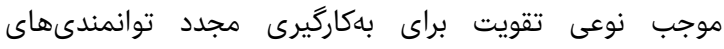

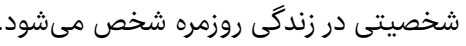

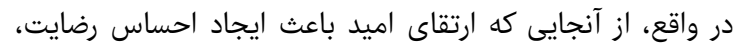

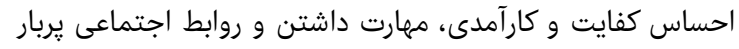

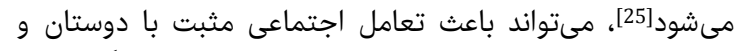

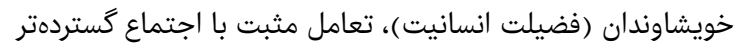

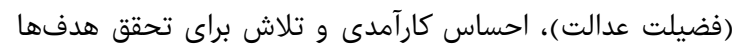

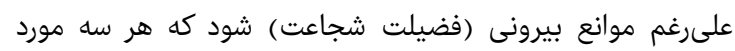

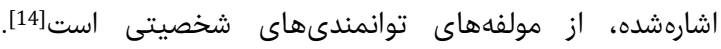

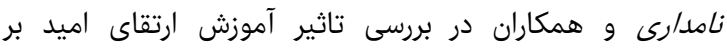

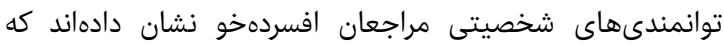

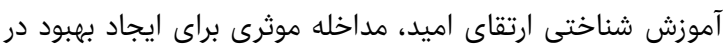

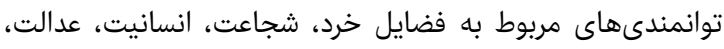
خويشتندارى و تعالى مراجعان بوده است [25].

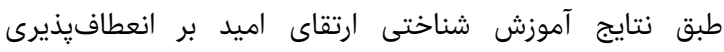

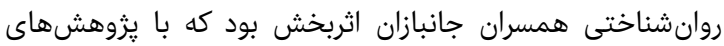

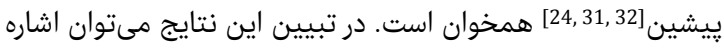

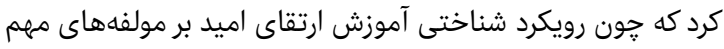

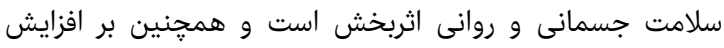

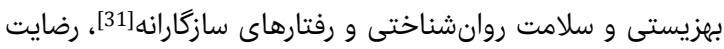

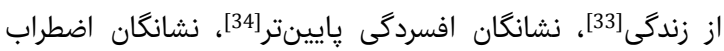

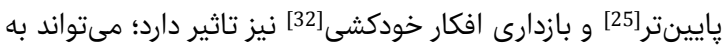

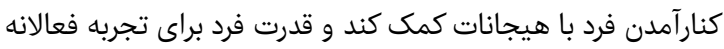

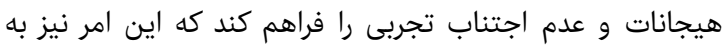

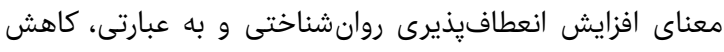
اجتناب تجربى است.

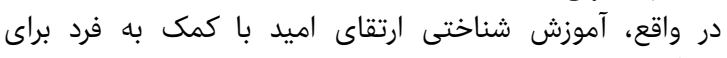

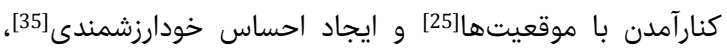

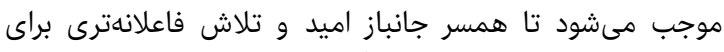
تجربه موقعيتهاى مختلف زندگى داشته باشد و هموان همواره بدون

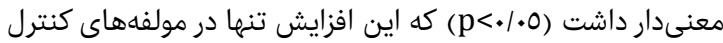

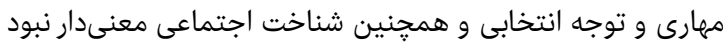

.$(\mathrm{p}>\cdot 1 \cdot 0)$

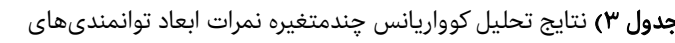

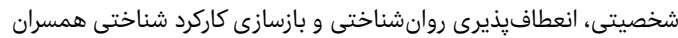

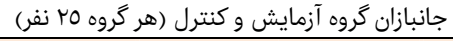

\begin{tabular}{|c|c|c|c|c|}
\hline سطح معنىدارى & F F F F F & يسآزمون & يِيش آزمون & متغيرها \\
\hline \multicolumn{5}{|c|}{ توانمندى شخصيتى } \\
\hline||$\cdots \mid$ & rN/qk & $\begin{array}{l}1 \mu q / q r \pm q / \varphi q \\
1 \% \cdot / . r+1 / v\end{array}$ & $1 r \cdot / \Delta \pm r N / 9 V$ & خرن \\
\hline \multirow{3}{*}{$\cdot|\cdots|$} & & 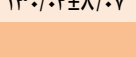 & $\pi T / \tau \cdot \pm / / \omega \lambda$ & شجاعت \\
\hline & \multirow{2}{*}{ HY/QS } & $1 . Y / . . \pm V / V V$ & $q \Delta / \mathcal{F} \wedge \pm \varepsilon / 1 \Lambda$ & آزمايش \\
\hline & & $94 / 9 \cdot \pm 0 / 94$ & $9 k / r \cdot \pm 0 / \wedge 9$ & كنترل \\
\hline \multirow{3}{*}{$.1 .+9$} & \multirow{3}{*}{$\Delta / I V$} & & & انسانيت \\
\hline & & $11 \cdot / q K_{ \pm} \mathrm{V} / .$. & $1.9 / 9 \Lambda \pm \varepsilon / 1 \vee$ & آزمايش \\
\hline & & $1.9 / \mu \cdot \pm 7 / 9 \pi$ & $1 \cdot \Delta / q \uparrow \pm \varepsilon / \Omega \varepsilon$ & كنترل \\
\hline \multirow{3}{*}{ 怆 } & \multirow{3}{*}{$k / q \mu$} & & & عدالت \\
\hline & & 1N/99 $\pm 7 / 10$ & $\wedge r / q 1 \pm 7 / \gamma)$ & 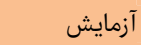 \\
\hline & & $\Lambda \kappa / \cdot V \pm V / \Lambda$. & $\wedge \mu / \& \Delta \pm 7 / q$. & كنترل \\
\hline \multirow{3}{*}{. $/ . r r$} & \multirow{3}{*}{ 1./VQ } & & & ميانهروى \\
\hline & & $৭ \wedge / \cdot \wedge \pm 0 / \wedge ৭$ & 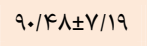 & آزمايش \\
\hline & & 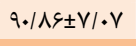 & $\Lambda \Lambda / F K_{ \pm} V / Y_{O}$ & كنترل \\
\hline \multirow{3}{*}{. $1 . \mathrm{rr}$} & \multirow{3}{*}{$\Delta / V V$} & & & تعالى \\
\hline & & $\Lambda V / / K \pm \Lambda / 97$ & $\Lambda r / \Lambda \varepsilon \pm Q / \varepsilon r$ & 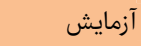 \\
\hline & & $\Lambda \mu / \cdot \varsigma \pm \Lambda / \vee O$ & $\Lambda \mu / \varepsilon r \pm q / \Lambda$. & كنترل \\
\hline \multirow{4}{*}{$\cdot|\ldots|$} & \multirow{3}{*}{$\mu k / F \mid$} & & \multicolumn{2}{|c|}{ انعطافيذيرى روانشناختى } \\
\hline & & $r \Delta / \& Y \pm I / \Lambda$. & $r \mu / V Y \pm r / \cdot r$ & آزمايش \\
\hline & & $r \mu / \Lambda \Lambda \pm 1 / 71$ & $r \mu / \Lambda K \pm 1 / 09$ & كنترل \\
\hline & & & & كاركرد شناختى \\
\hline \multirow{3}{*}{.$/ .4 k$} & \multirow{3}{*}{$Q / \Delta r$} & & & حافظه \\
\hline & & $11 / 9 Y \pm \varepsilon / 1 \Psi$ & $\Lambda / \varepsilon \psi_{ \pm} \psi / q$. & 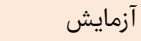 \\
\hline & & $\Lambda / \Lambda \Lambda \pm 79 / r$. & $\Lambda /|\varepsilon \pm \varepsilon / \varepsilon|$ & كنترل \\
\hline \multirow{3}{*}{.1 .9}$. & \multirow{3}{*}{$\mu / \cdot k$} & & \multicolumn{2}{|c|}{ كنترل مهارى و توجه انتخابى } \\
\hline & & $1 \cdot / \Lambda \cdot \pm 1 / \mu_{0}$ & $q / 9 \curlyvee \pm \varepsilon / 1$. & آزمايش \\
\hline & & $9 / \wedge K_{ \pm}+r / \cdot 0$ & $9 / 4 \wedge \pm r / 79$ & كنترل \\
\hline \multirow{3}{*}{||$\cdots \mid$} & \multirow{3}{*}{ GS/4S } & & & تصميمكيرى \\
\hline & & $\mathbb{I r / \Lambda \cdot \pm r / I \varepsilon}$ & $V / \mu \mu_{ \pm} r / 7 V$ & آزمايش \\
\hline & & $V / V Y \pm I / \varepsilon r$ & $V / \xi F_{ \pm} \mu / \mu$. & كنترل \\
\hline \multirow{3}{*}{$\cdot / . \bullet V$} & \multirow{3}{*}{$\Lambda / \cdot \Lambda$} & & & برنامهريزى \\
\hline & & $\Lambda / \mu q \pm r / . q$ & $\varepsilon / \digamma \wedge \pm \Psi / \varepsilon r$ & آزمايش \\
\hline & & $\varepsilon / V r \pm r / \mu r$ & $\varepsilon / \mu r \pm r / r \mu$ & كنترل \\
\hline & & & & توجه پايدار \\
\hline $.1 . * 4$ & $F(\Delta)$ & $V / \& \Lambda \pm \mid / 9 \vee$ & $8 / V Y \pm \Psi / 77$ & آزمايش \\
\hline 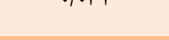 & & $\varepsilon / V \varphi \pm 1 / \mu q$ & $G / 9 r \pm r / r \mu$ & كنترل \\
\hline & & & & شناخت اجتماء \\
\hline . IDSK & . & $V / \Delta S \pm r / \cdot r$ & $V / \cdot \Lambda \pm I / r \Lambda$ & آزمايش \\
\hline •| $\mid$ QST & •/RT & $V / \cdot K_{ \pm} \mid / V q$ & \&/Q९土1// q & كنترل \\
\hline & & & ختى & انعطافيذيرى \\
\hline$\cdot \mid . .1$ & $|r / V|$ & $1 . / . r \pm r / \varepsilon r$ & 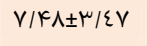 & آزمايش \\
\hline$\%$ & M/n & $V / \kappa \wedge \pm r / l$. & $V / \mu s \pm r / 09$ & كنترل \\
\hline
\end{tabular}

يزوهش حاضر با هدف بررسى اثربخشى آموزش شناختى ارتقاى

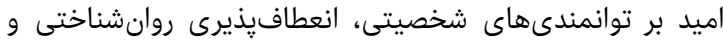

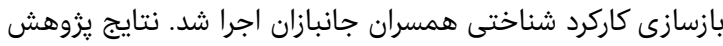

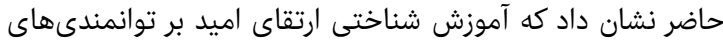

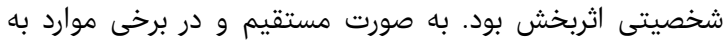

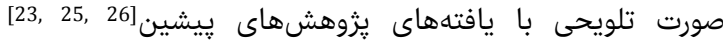




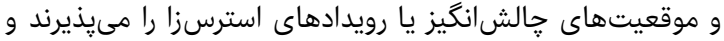

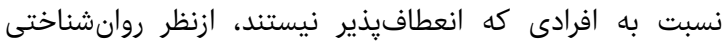

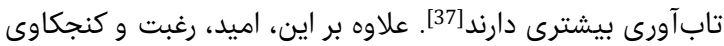

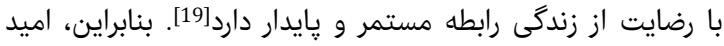

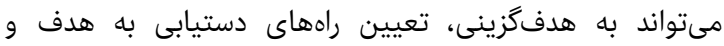

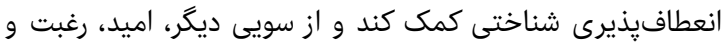

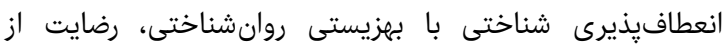

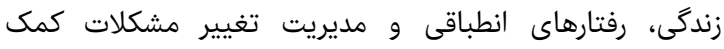

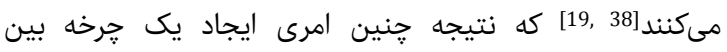

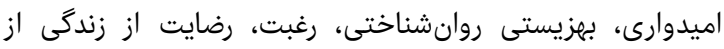

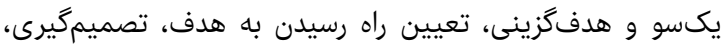

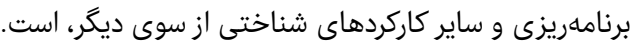

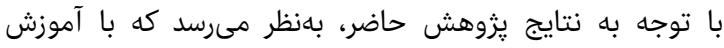

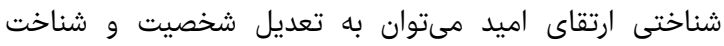

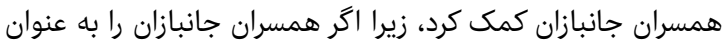

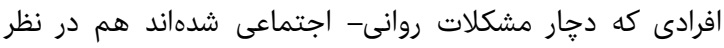

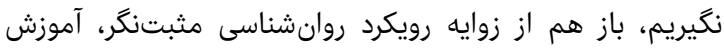

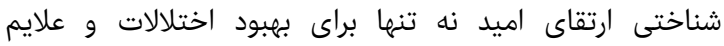
روانشناختى، بلكه براى ارتقا و شكوفايى شخصيتى و شناختى إنى اين إنائ كروه افراد مىتواند مفيد واقع شود.

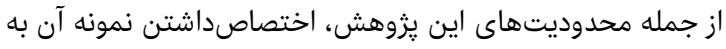

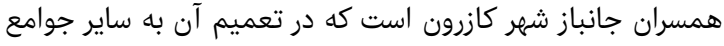

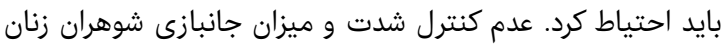

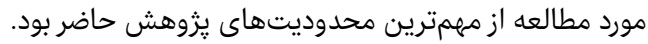

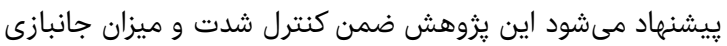

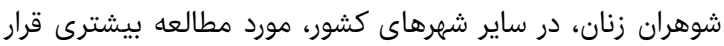

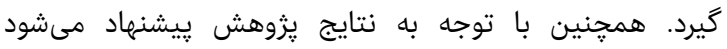

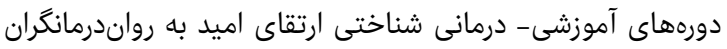

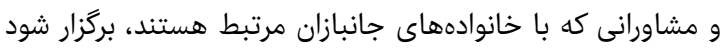

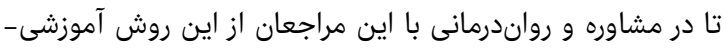

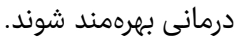

\section{نتيجه}

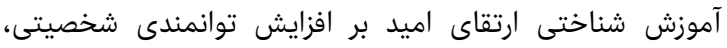

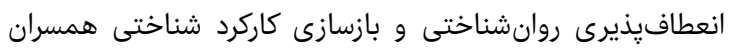

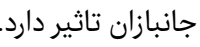

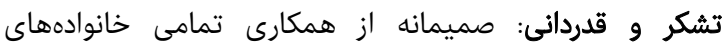
جانبازانى كه در اجراى اين يزوهش بـ بانئ يزوهشكران همكارى كردند،

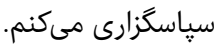

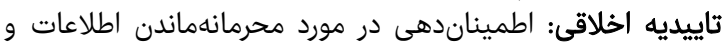

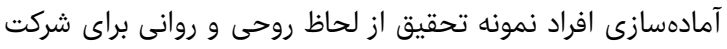

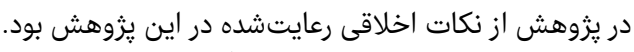

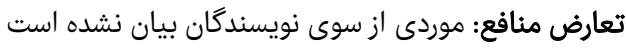

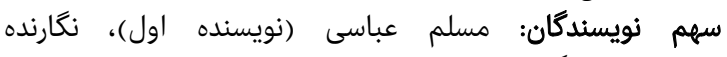

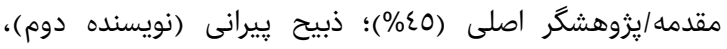

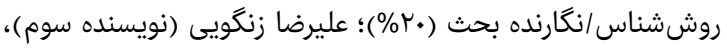

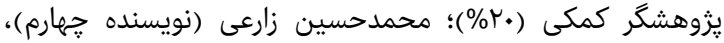

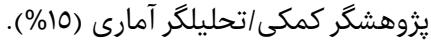
منابع مالى: اعتبار مالى اين يزوهش آماري توسط نويسندكان تامين شده

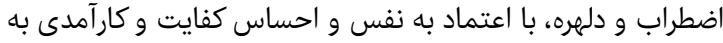

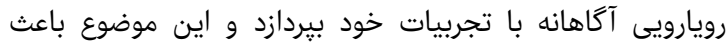

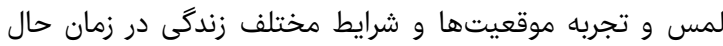

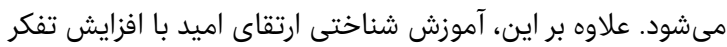

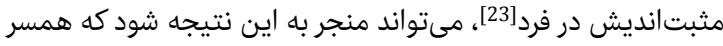

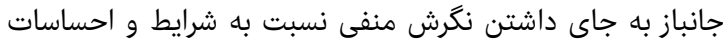

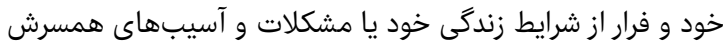

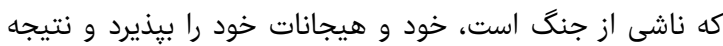

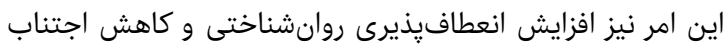

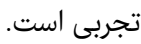
طبق يروتكل آموزش شناختى ارتقاى اميد، در اين شيوه آموزشى

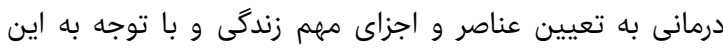

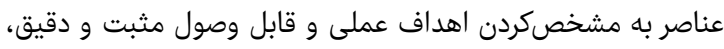

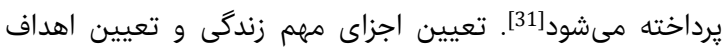

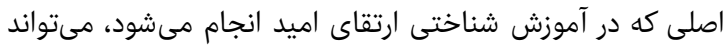

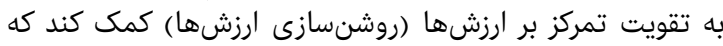

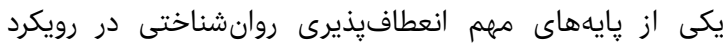

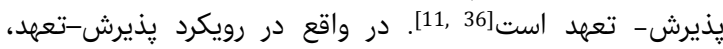

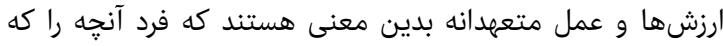

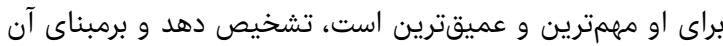

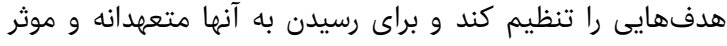

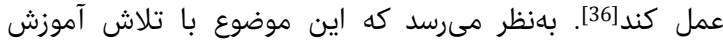

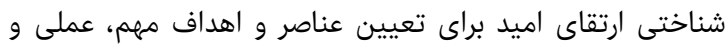

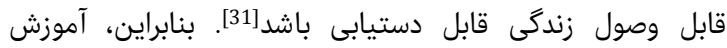

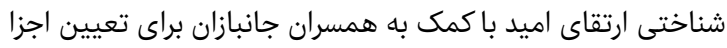

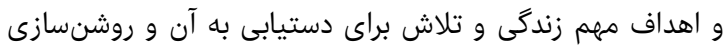

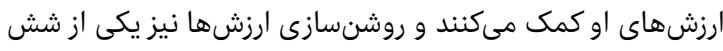

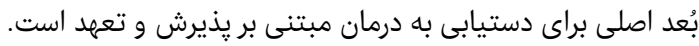

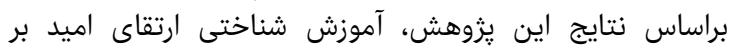

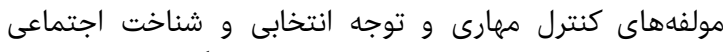

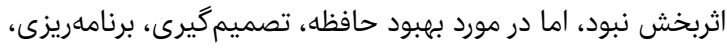

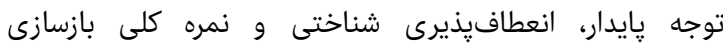

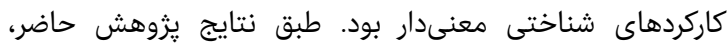

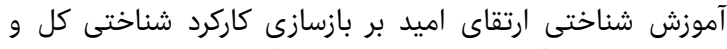

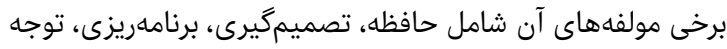

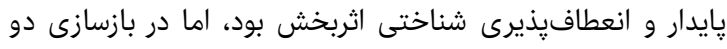

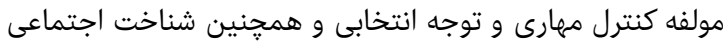

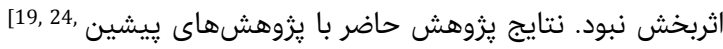

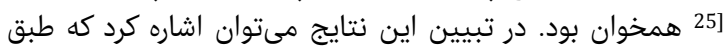

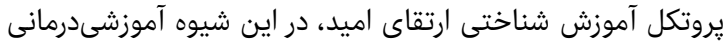

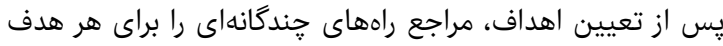

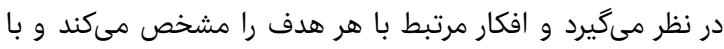

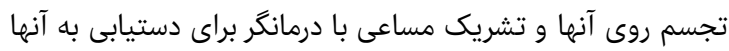

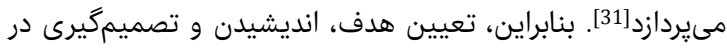

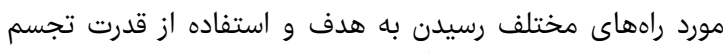

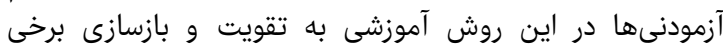

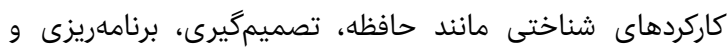

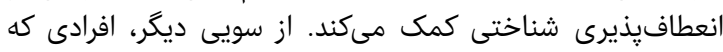

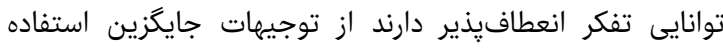

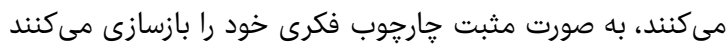


اثربخشى آموزش شناختى ارتقاى اميد بر توانمندىهاى شخصيتى، انعطافيذيرى روانشناختى و بازسازى كاركردهاى شناختى همسران جانبازان سرا

20- Snyder CR. Hope and depression: A light in the darkness. J Soc Clin Psychol. 2004;23(3):347-51.

21- Feldman DB, Snyder CR. Hope and the meaningful life: Theoretical and empirical associations betweengoaldirected thinking and life meaning. J Soc Clin Psychol. 2005;24(3):401-21.

22- Chang EC, DeSimone SL. The influence of hope on appraisals, coping, and dysphoria: A test of hope theory. J Soc Clin Psychol. 2001;20(2):117-29.

23- Steffen LE, Smith BW. The influence of between and within personhope among emergency responders on daily affect in a stress andcoping model. J Res Personal. 2013;47(6):738-47.

24- Fazeli M, Ehteshamzadeh P, Hashemi Sheikh Shabani $\mathrm{SE}$. The effectiveness of cognitive behavior therapy on cognitive flexibility of depressed people. Thought Behav Clin Psychol. 2015;9(34):27-36. [Persian]

25- Namdari K, Molavi H, Malekpour H, Kalantari M. The effect of cognitive hope enhancing training on character strengths of disthymic clients. J Clin Psychol. 2009;1(3):21-34. [Persian]

26- Hosseini SA, Badri R, Salimy H, Kolyaei L. The effect of cognitive hope enhancing training on reduction of victimization of high school students. Q educ Psychol. 2015;11(37):1-19. [Persian]

27- Delavar A. Theoretical and practical research in the humanities and social sciences. 2nd edition. Teharn: Roshd; 2001. [Persian]

28- Imani M. Investigating the factor structure of students unconscious flexibility questionnaire. J Teach Learn Stud. 2016;8(1):162-81. [Persian]

29- Nejati V. Cognitive abilities questionnaire: Development and evaluation of psychometric properties. Adv Cogn Sci. 2013;15(2):11-9. [Persian]

30- Snyder CR, Lopez ShJ, Shorey HS, Rand KL, Feldman DB. Hope theory, measurements, and applications to school psychology. Sch Psychol Q. 2003;18(2):122-39.

31- Rand KL, Martin AD, Shea AM. Hope, but not optimism predicts academic performance of law students beyond previous academic achievement. J Res Personal. 2011;45(6):683-6.

32- Tucker RP, Wingate LR, O'Keefe VM, Mills AC, Rasmussen $\mathrm{K}$, Davidson $\mathrm{CL}$, et al. Rumination and suicidal ideation: The moderating roles of hope and optimism. Personal Individ Differ. 2013;55(6):606-11.

33- Esteves M, Scoloveno RL, Mahat G, Yarcheski A, Scoloveno MA. An integrative review of adolescent hope. J Pediatr Nurs. 2013;28(2):105-13.

34- Berendes D, Keefe FJ, Somers TJ, Kothadia SM, Porter LS, Cheavens JS. Hope in the context of lung cancer: Relationships of hope to symptoms and psychological distress. J Pain Symptom Manage. 2010;40(2):174-82.

35- Alarcon GM, Bowling NA, Khazon S. Great expectations: A meta-analytic examination of optimism and hope. Person Individ Differ. 2013;54(7):821-7.

36- Harris R. Embracing your demons: An overview of acceptance and commitment therapy. Psychother Aust. 2006;12(4):2-8.

37- Haglund ME, Nestadt PS, Cooper NS, Southwick SM, Charney DS. Psychobiological mechanisms of resilience: Relevance to prevention and treatment of stress-related psychopathology.DevPsychopathol.2007;19(3):889-920. 38- Masuda A, Tully EC. The role of mindfulness and psychological flexibility in somatization, depression, anxiety, and general psychological distress in a nonclinical college sample. J Evid Base Complement Altern Med. 2012;17(1):66-71.
1- Dekel R, Monson CM. Military-related post-traumatic stress disorder and family relations: Current knowledge and future directions. Aggress Violent Behav. 2010;15(4):303-9.

2- Saleh S, Zahedi Asl M. Correlation of social support with social health of psychiatry veterans wives. Iran J War Public Health. 2014;6(5):201-6. [Persian]

3- Ghahari Sh, Mehryar AH, Birashk B. Comparitive study of some mental disorders in children of fatherless, martyred's, veteran's, and normal (7-12 years old) in Chalus city. J Mazandaran Univ Med Sci. 2003;13(41):8191. [Persian]

4- Afrooz GH, Vysmeh AA. The relationship between depression and how veterans sons with their fathers. J Psychol Educ Sci. 2001;31(2):35-50. [Persian]

5- Molaee M, Moradi A, Gharayee B. Executive function and neuropsychological evidence among OCD and GAD. J Behav Sci. 2008;1(2):131-41. [Persian]

6- Ghamari Givi H, Maqsoud N, Dehghani F. The effect of cognitive rehabilitation on the reconstruction of executive functions of obsessive-compulsive patients. Q Clin Psychol. 2014;4(16):101-28. [Persian]

7- Crone EA, Wendelken C, Donohue SE, Bunge SA. Neural evidence for dissociable components of taskswitching. Cerebral Cortex. 2006;16(4):475-86.

8- Alemi S, moradi A, Jonabian A. The psychological (emotional and cognitive) performance of spouse's cancer during and after treatment. J Res Psychol Health. 2012;6(1):36-44. [Presian]

9- Pourfaraj Omran M. The effectiveness of acceptance and commitment group therapy in social phobia of students. Knowl Health. 2011;6(2):1-5. [Persian]

10- Hayes SC, Luoma JB, Bond FW, Masuda A, Lillis J. Acceptance and commitment therapy: Model, processes and outcomes. Behav Res Ther. 2006;44(1):1-25.

11- Hayes SC, Strosahl KD, Wilson KG. Acceptance and commitment therapy: An experiential approach to behavior change. New York: Guilford Publication; 1999.

12- Fledderus M, Bohlmeijer ET, Pieterse ME. Does experientialavoidance mediate the effects of maladaptive coping styles onpsychopathology and mental health? Behav Modif. 2010;34(6):503-19.

13- Westin V, Hayes SC, Andersson G. Is it the sound or your relationship to it? The role of acceptance in predicting tinnitus impact. Behav Res Ther. 2008;46(12):1259-65.

14- Karimizadeh F, ShafiAbadi A, Farahbakhsh K. The investigate the relationship between personality abilities and the moral foundations of delinquents juvenile. Q Psychol Educ Sci. 2016;2(2):11-21. [Persian]

15- Li T, Liu X. Role of character strengths and stress in psychological symptoms among chinese secondary vocational school students. Psychology. 2016;7(1):52-61. 16- Seligman ME, Steen TA, Park N, Peterson C. Positive psychology progress: Empirical validation of interventions. Am psychol. 2005;60(5):410-21.

17- Peterson Ch, Ruch W, Beermann U, Park N, Seligman MEP. Strengths of character, orientations to happiness, and life satisfaction. J Posit Psychol. 2007;2(3):149-56.

18- Kozlova NV. The psychological and acmeological approach in an educational practice of the higher school. Siberian J Psychol. 2008;29:79-85. [Russian]

19- Park N, Peterson C, Seligman MEP. Strengths of character and well-being. J Soc Clin Psychol. 2004;23(5):603-19. 